\title{
Girolamo Tiraboschi faiseur et défaiseur de héros dans la Storia della letteratura italiana (1772)
}

Eroi e no nella Storia della letteratura italiana di G. Tiraboschi

Heroic and non-heroic writers in the Storia della letteratura italiana by

G. Tiraboschi

\section{Philippe Simon}

\section{OpenEdition}

Journals

Édition électronique

URL : http://journals.openedition.org/cei/1036

DOI : 10.4000/cei.1036

ISSN : 2260-779X

\section{Éditeur}

UGA Éditions/Université Grenoble Alpes

\section{Édition imprimée}

Date de publication : 31 octobre 2012

Pagination : 199-209

ISBN : 978-2-84310-234-9

ISSN : 1770-9571

\section{Référence électronique}

Philippe Simon, «Girolamo Tiraboschi faiseur et défaiseur de héros dans la Storia della letteratura italiana (1772)», Cahiers d'études italiennes [En ligne], 15 | 2012, mis en ligne le 30 avril 2014, consulté le 27 mars 2021. URL : http://journals.openedition.org/cei/1036 ; DOI : https://doi.org/10.4000/cei. 1036 


\title{
GIROLAMO TIRABOSCHI FAISEUR ET DÉFAISEUR DE HÉROS DANS LA STORIA DELLA LETTERATURA ITALIANA (1772)
}

\author{
Philippe Simon \\ Université de Paris-Sorbonne
}

La qualité, le terme même de héros ont une très longue histoire qui remonte à l'Antiquité, en particulier gréco-latine. Les poètes, les premiers, Homère, Hésiode, Ovide ou Virgile évoquent tout d'abord des personnages mythiques, mi-hommes mi-dieux, aux qualités exceptionnelles comme Achille, Hercule ou Énée parmi tant d'autres. La figure héroïque, par la suite, s'humanise et se laïcise chez Plutarque et Cornelius Nepos qui décrivent des souverains, de grands capitaines ou des orateurs qui se distinguent par leurs exploits, leur force de caractère ou d'autres traits remarquables. Les héros retrouvent des caractéristiques divines et surhumaines dans les hagiographies chrétiennes du Moyen Âge dont l'un des plus célèbres recueils est la Légende Dorée de Jacques de Voragine (I26I). Puis, progressivement l'héroïsme s'ouvre à d'autres "corporations" et se "nationalise» voire se "régionalise». Ainsi Giorgio Vasari dans ses Vite de' più eccellenti architetti, pittori, et scultori italiani (I550) présente des artistes italiens qui se distinguent, avec une préférence partisane pour les toscans. Bref, petit à petit, le héros peut appartenir à des catégories sociales et professionnelles de plus en plus larges ${ }^{\mathrm{I}}$. Selon la définition plutôt générale qu'en donne le Vocabolario degli Accademici della Crusca dès sa deuxième édition de 1623 , il est simplement assimilé à un "huomo illustre».

$\mathrm{Au} \mathrm{XVII}{ }^{\mathrm{e}}$ siècle, justement, fleurissent, spécialement en Italie, des répertoires généraux qui présentent, un peu dans le désordre et avec des critères

I. Sur les héros et le concept d'héroïsme, voir La fabrique des héros sous la direction de P. Centlivres, D. Fabre et F. Zonabend, textes réunis par C. Voisenat et E. Julien, Paris, Édition de la Maison des sciences de l'homme, collection "Ethnologie de la France, Cahier n ${ }^{\circ}$ I2", 1999, XI-318 p., et J. Campbell, Le héros aux mille et un visages, traduit de l'anglais [The hero with a thousand faces] par $\mathrm{H}$. Crès, Paris, Oxus, 2010, $4 \mathrm{IO} \mathrm{p}$. 
plutôt subjectifs, des centaines d'illustres contemporains italiens et étrangers. Sont ainsi honorées dans de brèves notices individuelles, toutes à peu près d'égale longueur et sur un plan de quasi-égalité, carrières, œuvres, qualités exceptionnelles de grands prélats, poètes, savants, historiens. Parmi les principaux ouvrages de cette nature, on peut citer ou le Musaeum Historicum et Physicum de Giovanni Vincenzo Imperiali (I640), la Pinacotheca de Giovanni Vittorio Rossi (I643) qui connaît une large diffusion européenne ou encore le Teatro d'huomini letterati de Girolamo Ghilini (I647). Dans les galeries de portraits un peu stéréotypés et répétitifs qu'ils proposent, on ne peut pas dire que se détachent de véritables héros. Rossi, par exemple, se plaît aussi à souligner tel ou tel travers de ceux qu'il présente ${ }^{2}$.

$\mathrm{Au} \mathrm{XVIII}{ }^{\mathrm{e}}$ siècle apparaissent des ouvrages plus spécialisés et précis qui se proposent de répertorier tout ou partie des écrivains italiens. L'Istoria della volgar poesia de Giovanni Mario Crescimbeni ${ }^{3}$ ne présente que des poètes. L'auteur cherche bien à organiser chronologiquement et à classer sa matière pour mettre en exergue "poeti più cogniti», mais il entend simplement, selon ses propres termes, "dir solo quanto basta per far vedere lo stato della Volgar Poesia ${ }^{4}$. Ainsi, après des considérations générales sur l'évolution de la poésie, Crescimbeni présente des fiches biographiques plus ou moins précises consacrées à une série de poètes du XIII ${ }^{\mathrm{e}}$ au XVIII ${ }^{\mathrm{e}}$ siècle, complétées par les appréciations critiques de "savi letterati» sur leurs œuvres. S'ajoutent encore, pour certains d'entre eux, des morceaux choisis illustrant leur style poétique. Enfin sont simplement cités une longue série de rimatori, poètes mineurs, $\mathrm{du} \mathrm{XIII}{ }^{\mathrm{e}}$ au $\mathrm{XVIII}^{\mathrm{e}}$ siècle. Bref, il existe une certaine volonté au moins implicite de classer les poètes : les fiches qui sont consacrées à certains des plus illustres d'entre eux sont plus ou moins précises et, comme nous l'avons dit, seuls certains poètes ont droit de surcroît à un appendice bibliographique et à des morceaux choisis. D'autre part, le critère de "significativité» dans le choix des personnages apparaît encore une fois assez subjectif. Enfin, Crescimbeni ne souhaite pas "tesser vite né elogi storici». La tonalité des développements consacrés aux poètes n'est donc nullement héroïque. Dans l'Idea della storia dell'Italia letterata, Giacinto Gimmas indique bien, en quelques mots, les mérites de tel ou tel écrivain mais, plutôt que de mettre en exergue les individus

\footnotetext{
2. Voir G. Getto, Storia delle storie letterarie, Florence, Sansoni, I98I p. 25-27.

3. G. M. Crescimbeni, Istoria della volgar poesia, Rome, Rossi, I7I4.

4. Ibid., "Introduzione» n.n.

5. G. Gimma, Idea della storia dell'Italia letterata, Naples, Mosca, I723.
} 
illustres, il présente plutôt les genres de la littérature ou, en fait, de la culture italienne et il est bien difficile de s'y retrouver dans cet ouvrage riche d'informations, certes, mais touffu et chaotique. Enfin, Giammaria Mazzuchelli dans ses Scrittori d'Italia (I753) ${ }^{6}$ avait l'ambition de produire un dictionnaire exhaustif d'écrivains italiens. Certes, la plus ou moins grande longueur des notices permet implicitement de déceler les auteurs illustres que l'auteur a cherché à mettre en avant mais, plus que leurs éventuelles qualités ou caractéristiques héroïques, sont plutôt détaillées leurs biographies ainsi qu'une liste la plus complète possible de leurs ouvrages. Cependant, G. M. Mazzuchelli n'a réussi à traiter que les deux premières lettres de l'alphabet, il est donc très fragmentaire.

En réalité, la première véritable histoire complète de la littérature nationale italienne est la Storia della letteratura italiana (1772) de Girolamo Tiraboschi, jésuite érudit, bibliothécaire du duc de Modène ${ }^{7}$. Même s'il puise et tire forcément d'utiles suggestions et informations des ouvrages précédents, Tiraboschi ne se reconnaît aucun maître et s'attribue, non sans quelque raison, le mérite d'avoir été le premier à avoir eu l'idée d'écrire une histoire de la littérature italienne ${ }^{8}$. Dans son monumental ouvrage, l'auteur se propose de suivre les différents développements de la littérature, c'est-à-dire en fait de la culture italienne (conformément à l'acception du terme à l'époque) depuis les temps les plus reculés, étrusques en l'occurrence, jusqu'au XVII ${ }^{\mathrm{e}}$ siècle dans une perspective plus géographique que linguistique.

L'ouvrage s'ouvre sur une tonalité héroïque. L'histoire de la littérature italienne est regardée comme une sorte d'épopée :

Non vi ha Scrittore alcuno imparziale e sincero, che alla nostra Italia non conceda volentieri il glorioso nome di Madre e Nudrice delle Scienze, e delle Bell'Arti. II favore, di cui esse hanno tra noi goduto, e il fervore, con cui da' nostri si son cultivate e ne' più lieti tempi del Romano Impero, e ne felici secoli del loro risorgimento, le ha condotte a tal perfezione, e a tal onore le ha sollevate, che gli stranieri, e quegli ancora tra essi, che della lor gloria son più gelosi, sono astretti a confessare, che da noi mosse primieramente quella si chiara luce, che baleno a' loro sguardi, e che gli scorse a veder cose ad essi finallora ignote,

6. G. M. Mazzuchelli, Gli scrittori d'Italia, Brescia, Giambatista Bossini, 1753-1763, 6 vol.

7. Nous avons utilisé G. Tiraboschi, Storia della letteratura italiana, Florence, Molini e Landi, I805-I813, 9 volumes. Toutes les notes renvoient à cette édition, désormais citée comme Storia... Pour une présentation globale de l'ouvrage, voir M. Mari, Il genio freddo: la storiografia letteraria di Girolamo Tiraboschi nuova edizione accresciuta, Milan, CUEM, I999, $432 \mathrm{p}$.

8. G. Tiraboschi, Storia..., ouvr. cité, vol. I, p. xxvır. 
lit-on dans les premières lignes de la Préface de la Storia ${ }^{9}$. Cette épopée, certes, connait des hauts et des bas. Elle passe par des phases de "progressi, decadenza, risorgimento ${ }^{\mathrm{IO}}$ " dont Tiraboschi veut suivre les vicissitudes.

La présentation de la littérature est très structurée : quelques pages après l'envolée lyrique du début, nous en sont détaillées les caractéristiques. Dans le tableau global qu'il entend brosser de chaque siècle, Tiraboschi explique qu'il illustrera tout d'abord « $i$ mezzi che giovarono a coltivare le scienze", c'est-à-dire le contexte culturel et intellectuel dans lequel les écrivains ont pu s'épanouir ${ }^{\text {II }}$. Il précise ensuite qu'il ne parlera pas de tous les auteurs italiens mais seulement de certains d'entre eux en fonction de leurs mérites. Le premier, il les répartit en trois catégories cohérentes distinguant "coloro che salirono a maggior fama e che col sapere e le opere si renderono più illustri», ceux qui "non furono per ugual modo famosi», et enfin ceux dont "mi basterà accennare $i$ nomi $i^{\mathrm{I} 2}$ ». D' autre part, Tiraboschi indique bien vouloir parler exclusivement d'écrivains italiens et revendique de ne pas devoir «da lor medesimi [gli stranieri] apprendere le nostre lodi' ${ }^{13}$ ".

Dans cette histoire de la littérature italienne, Tiraboschi se propose donc bien de classer les écrivains qu'il va présenter en fonction de critères de «significativité» précis basés classiquement sur les jugements des lettrés, si possible contemporains des auteurs qu'ils évaluent. Il se réserve toutefois de les contredire ${ }^{14}$. Parfois également, il tire de l'ombre un écrivain oublié dont il glorifie les mérites. Ainsi, au milieu d'un grand nombre d'inconnus ou d'auteurs mineurs, il met en exergue des grands, des héros, des hommes illustres ayant marqué la littérature italienne. Tiraboschi utilise d'ailleurs, de loin en loin et de siècle en siècle, le terme même de héros appliqué de façon assez surprenante à des personnalités aussi différentes que Manfredi, Cola di Rienzo, et Lorenzo de' Medici ou encore à un navigateur comme Cristoforo Colombo, à des militaires comme Alessandro Farnese ou encore Ferdinando d'Avalos, marchese di Vasto, également grand mécène. Cela dit, il utilise également d'autres qualificatifs pour distinguer ceux des écrivains qui se sont particulièrement, héroïquement, distingués. Enfin, pour permettre au lecteur d'identifier plus facilement les auteurs les plus illustres sur lesquels il entend insister — les héros -

9. Ibid., vol. I, p. xxv.

Io. Ibid., vol. I, p. xxix.

II. Ibid., vol. I, p. xxx.

I2. Ibid., vol. I, p. Xxix.

13. Ibid., vol. I, p. Xxvi.

I4. Ibid., vol. I, p. xxxiv. 
leurs noms sont cités en marge du texte et ils sont également mentionnés dans la table des matières très précise, par genres, qui est présente dans la Storia à partir de la deuxième édition de Modène en 1784 . Mais ces écrivains illustres, ainsi distingués typographiquement, ne sont pas tous sur le même plan. C'est justement ce classement, cette typologie des héros littéraires chez Tiraboschi que nous allons essayer d'illustrer ici à propos du XVII ${ }^{\mathrm{e}}$ siècle, une époque à propos de laquelle il est un des premiers à exprimer un jugement d'ensemble particulièrement contrasté.

Tout d'abord, n'est pas héros qui veut, et Tiraboschi remet parfois radicalement en cause les jugements des contemporains sur certains auteurs du XVII ${ }^{\mathrm{e}}$ siècle. Le cas le plus significatif à cet égard est celui de Giovanni Battista Marino, véritable coqueluche de son temps à qui "in ogni parte [...] tributavansi [onori] » et dont "favellano tanti altri scrittori», mais que Tiraboschi définit comme "il maggior corrompitor del gusto italiano ${ }^{15}$ ". S'arrêtant sur la vie du poète, il souligne le succès qu'il remporte auprès de grands personnages, notamment Charles Emmanuel de Savoie duquel "ottenne tal grazia [...] che questi lo ascrisse all'ordine dei SS Maurizio e Lazzaro e fermollo alla sua corte collo [...] carattere di segretario", de Marie de Médicis encore "da cui ebbe un'annua pensione». Il ne s'agit là que d'honneurs matériels finalement peu glorieux. Insignifiantes aussi sont les âpres querelles littéraires, considérées comme d'importance fondamentale à l'époque, qui se déchaînent à la publication de ses ouvres principales et que Tiraboschi ramène ironiquement à leurs véritables proportions. Ainsi, il rappelle, non sans esprit, que dans un de ses sonnets Marino "prese un solenne granchio confondendo il leone da Ercole ucciso con l'idra lernea" ajoutant aussitôt qu'il s'agissait d'un "oggetto, a dir vero, di troppo lieve momento perché si dovesser per esso pubblicar tanti libri ${ }^{16}$ ". Quant aux œuvres de Marino, elles sont ou scandaleuses ou «troppo famose» comme l'Adone, son poème le plus célèbre. Pour finir, la comparaison même de ce poète avec Icare, en fin de notice, montre qu'il avait bien des velléités d'héroïsme et de gloire mais "per farsi più grande divenne mostruoso", et son exemple «fu dannoso all'Italia ${ }^{17}$ ». Bref, pour Tiraboschi, Marino est un des faux héros de l'époque et il entraîne dans sa chute d'autres poètes comme Claudio Achillini, sévèrement jugé lui aussi dans la Storia : "gli elogi $[\mathrm{da}$ lui] ricevuti son ben compensati dall'abbandono in cui or se ne giacciono le

I5. Ibid., vol. VIII, p. 452.

I6. Ibid., vol. VIII, p. 453 .

I7. Ibid., vol. VIII, p. 454-456. 
opere $^{18}$. " On remarque en tout cas que Tiraboschi accorde à Marino, au moins quantitativement, une assez grande attention. Il s'agit donc bien pour lui d'un personnage important dans son siècle auquel il convient, même si c'est pour en dénoncer les tares, de faire une place significative. De la même façon, Tiraboschi donne un certain relief à quelques héros «manqués» bien connus de leur temps sans doute plutôt pour des raisons anecdotiques, car il s'agit de figures aux caractéristiques surprenantes, tragiques ou plus sulfureuses. Le cas du jésuite Teofilo Rainaudo, auquel sont consacrés d'assez longs développements, prête plutôt à sourire : le nom de ce dernier en effet, nous dit Tiraboschi, serait «assai più glorioso ne' fasti della letteratura se avesse voluto ristringersi a minor numero di opere e se avesse saputo frenar la sua troppo focosa indole ${ }^{19}$ ". Ce "héros», il se plaît à en suivre la vie soulignant son "illibata pietà" ses "severi costumi», son "ardente zelo", mais aussi sa "mordacità e [...] asprezza che ad un uomo dotto e molto più ad un religioso sembra mal convenisse ${ }^{20}$ ", et surtout sa graphomanie. Pour conclure, Tiraboschi s'effraie justement de l'étendue des ouvres de Rainaudo: "[...] ma come potrò io dare in breve l'idea di tutte queste opere? Novantatre ne annovera il p. Niceron ed esse sono comprese in diciannove tomi in folio." Il ajoute encore, non sans humour, que la publication de cette masse d'ouvrages fit que le libraire qui s'en était chargé et "credeva di adunarne tesori andò fallito ${ }^{2 \mathrm{I}}$ ". Le seul "héroïsme» burlesque de cet étrange personnage ne serait donc pas du tout lié au contenu de ses œuvres mais à son invraisemblable fécondité.

Plus tragique en revanche est le destin de Virginio Cesarini, homme de «vastissima erudizione» que Tiraboschi évoque rapidement avec des mots sobres et pleins d'émotion : "uguale e forse maggior gloria poteva la poesia latina aspettarsi da $V$. Cesarini di nobilissima famiglia romana se un'immatura morte non l'avesse rapito ${ }^{22}$ ».

Enfin, beaucoup plus troublant est le cas de Marcantonio de Dominis, jésuite, archevêque de Split et théologien auquel Tiraboschi consacre une longue notice. Il possède les potentialités qui auraient pu en faire un héros : il fait preuve d'un "grande ingegno meritevole d'immortal fama", "molti scrittori ragionano di lui»; il est issu "di un'antica e illustre famiglia» et "in età fanciullesca diede buon saggio di sé». Au niveau professionnel aussi, il se fait honorablement remarquer : "lesse con gran concorso

\footnotetext{
I8. Ibid., vol. VIII, p. 460.

19. Ibid., vol. VIII, p. 156.

20. Ibid., vol. VIII, p. I56-I58.

2I. Ibid., vol. VIII, p. 158 .

22. Ibid., vol. VIII, p. 5 IO.
} 
le matematiche in Padova". Toutefois, "in mezzo ai lieti saggi ch'ei dava del suo talento scorgevasi in lui uno spirito torbido apmbizioso inquieto e insofferente di giogo». C'est donc ces défauts de constitution intellectuelle et caractérielle qui l'empêchent de mériter "l'immortal fama» et le poussent au contraire dans les bras des hérétiques. Et on sent le trouble de Tiraboschi qui détaille les péripéties de l'itinéraire doctrinal et humain de l'archevêque dévoyé de Split de la Suisse à la Grande Bretagne jusqu'au repentir final devant le pape Grégoire XV lui-même. Mais tout n'est pas bien qui finit bien, car "essendosi comprovato chegli era veramente ricaduto nell'eresia il corpo ne fu poscia dato alle fiamme ${ }^{23}$ ", une fin tout sauf hérö̈que.

Les cas de ces faux héros ou de ces héros "manqués» ne sont pas très nombreux ni originaux. Tiraboschi rassemble et condense des informations provenant la plupart du temps de sources précédentes plus qu'il n'innove, mais il les met en ordre et les classe.

Les plus nombreux à être mis à l'honneur, quelques dizaines pour le $\mathrm{XVII}^{\mathrm{e}}$ siècle, sont une longue série d' "uomini illustri», un peu ternes, pas forcément héroïques, ni par leurs vies ni par leurs œuvres mais tout de même remarquables par telle ou telle caractéristique. Les dits personnages sont désignés suivant les cas de façon un peu stéréotypée comme "dotti», "eruditi», "grandi» ou "illustri» sans plus. Des termes que l'on retrouve par exemple dans l'assez court éloge consacré au Cardinal Giovanni Bona, savant illustrateur de la liturgie catholique, défini comme «dotto non men che pio scrittore alienissimo dalle dignità ${ }^{24}$ » auxquelles il préfère ses chères études. Bref un bon et sérieux lettré dont on trouve un assez grand nombre d'exemples parmi les écrivains religieux, comme également Monsignor Giovanni Ciampini, historien ecclésiastique "dotto scrittore» dont la vie "non ci offre cose degne di particolar riflessione "2, ou le cardinal Arrigo Noris "dottissimo scrittore ${ }^{26}$ " Certains laïques aussi rentrent dans cette même catégorie comme l'astronome Geminiano Montanari «il più illustre [...] degli astronomi di secondo ordine ${ }^{27}$ ", ou l'historien de la littérature Gian Vittorio Rossi qui mérite une "più distinta menzione ${ }^{28}$ ". Pour cette catégorie encore, Tiraboschi reprend le plus souvent des informations

23. Ibid., vol. VIII, p. Io6-Io9 (pour l'ensemble des citations).

24. Ibid., vol. VIII, p. I2O.

25. Ibid., vol. VIII, p. I25.

26. Ibid., vol. VIII, p. I4O.

27. Ibid., vol. VIII, p. 236.

28. Ibid., vol. VIII, p. 427. 
puisées soit dans des répertoires spécialisés soit dans des biographies qu’il condense et ordonne.

Certains autres écrivains, en revanche, se distinguent un peu davantage : les termes employés à leur égard sont en effet plus élogieux. C’est le cas, parmi d'autres, d'Enrico Caterino Davila. Dans l'éloge que Tiraboschi en donne, il le qualifie "d'uno dé più illustri storici del tempo ${ }^{29}$ ". Il est en cela bien supérieur à la longue série d'autres historiens plus ou moins obscurs dont Tiraboschi raille, à un autre endroit, la quantité, en particulier dans l'État pontifical ${ }^{30}$. L'œuvre majeure de Davila, la Storia delle guerre civili di Francia (1630), a droit a "una magnifica edizione» et, si elle n'est pas exempte de défauts, "per confessione degli stessi francesi essa è una delle migliori storie che quelle guerre abbiano avuto». Cela dit, la vie de cet historien n'est pas franchement héroïque non plus. En fait, Tiraboschi s'apitoie plutôt sur ses mésaventures et, en particulier, sur sa mort tragique ${ }^{31}$. Quelques savants également sont qualifiés de façon très flatteuse : comme Evangelista Torricelli, "uno de più grandi uomini del secolo ${ }^{32}$ ", ou encore Giandomenico Cassini, "gran genio ${ }^{33}$ ", ou enfin Marcello Malpighi, «uno dé più rari geni ${ }^{34}$ ». Il n'y a rien d'original non plus dans ces jugements qui, la plupart du temps, reprennent les opinions proposées dans d'autres répertoires, comme celui de Rossi ou de Mazzuchelli, ou dans des biographies précédentes.

Dans certains cas pourtant Tiraboschi innove et cherche à réévaluer certaines figures laissées dans l'ombre comme celle de Giacomo Puricelli "uomo veramente dottissimo", une des qualités majeures de cet historien milanais. Ce dernier a droit a un éloge appuyé. Tiraboschi souligne son "vivo ingegno", son "instancabile avidità di apprendere cose nuove", le courage aussi dont il fait preuve pour secourir les malades lors de l'épidémie de peste de I630, sa fécondité hors du commun ${ }^{35}$ et l'extrême qualité de ses recherches sur la Basilique ambrosienne de Milan. Pour finir, il rend hommage à ce grand homme comme à un maître «delle fatiche del quale io mi son giovato per comporre la mia opera». Bref, Puricelli ouvre la voie aux érudits par ses travaux sur Milan et son nom "rimarrà immortale e glorioso presso i saggi estimatori della vera erudizione ${ }^{36} »$.

29. Ibid., vol. VIII, p. $4 \mathrm{I} 3$.

30. Ibid., vol. VIII, p. 397.

3I. Ibid., vol. VIII, p. $4 \mathrm{I} 3$.

32. Ibid., vol. VIII, p. 204.

33. Ibid., vol. VIII, p. 256.

34. Ibid., vol. VIII, p. 306.

35. Ibid., vol. VIII, p. 405.

36. Ibid., vol. VIII, p. 406-407. 
Une des seules figures véritablement héroïques, dans la mesure où elle se place vraiment au-dessus du commun des mortels, où elle constitue un modèle par sa vie, sa carrière, son apport culturel dans la mesure, enfin, où elle suscite de l'admiration universelle, est sans aucun doute celle de Galileo Galilei. Même s'il n'a pas droit à des égards typographiques particuliers par rapport aux autres personnalités auxquelles nous avons fait allusion, en consultant la table des matières, on se rend compte immédiatement que de nombreuses pages lui sont consacrées. Dès les premières lignes du long éloge qui lui est consacré, il est explicitement opposé à Tommaso Campanella qui représenterait un autre de ces héros manqués auxquels nous avons déjà fait allusion. Alors que ce dernier

co'suoi ingegnosi deliri sconvolgeva la filosofia tutta senza rischiararne gli astrusi misteri il gran Galileo con passo franco e sicuro innoltravasi arditamente nel vasto e sconosciuto regno della natura, e tanti trofei v'innalzava all'immortalità del suo nome quante vi facea ad ogni passo nuove e meravigliose scoperte ${ }^{37}$.

La tonalité est clairement héroïque. Galilée est un grand dont il convient de bien retracer la geste à la suite de ses nombreux biographes et panégyristes comme Vincenzo Viviani, Angelo Fabroni, Juan Andrès et d'autres. Parmi les composantes implicites qui contribuent à former son génie, figure sa noblesse d'extraction : Tiraboschi souligne sur la foi d' "autentici documenti " qu'il cite que Galilée est fils d'un "nobile fiorentino", balayant ainsi l'hypothèse infamante d'une naissance illégitime colportée entre autres par l'Encyclopédie de d'Alembert et Diderot pour laquelle d'ailleurs Tiraboschi n'a guère de sympathies et dont il ne manque pas de souligner les erreurs. Hors du commun sont aussi les talents naturels de Galilée qui "poco dovette ai maestri e quasi tutto a se stess $0^{38}$ " et est véritablement un homme complet doué aussi bien pour le dessin que pour la musique, la poésie et les sciences. Dans la suite de la notice, on trouve encore des accents dignes d'une épopée un peu tragique : d'un côté, Galilée fait une brillante carrière "onorato da tutti distinto con ampli privilegi 39 " mais, compte tenu de son avance intellectuelle, des obstacles se dressent devant lui, en particulier ecclésiastiques. Et Tiraboschi s'arrange comme il peut entre son désir de tisser l'éloge de Galilée et sa volonté de minimiser l'attitude négative de l'Église (et notamment de la Compagnie de Jésus à laquelle il appartient) envers lui. Il souligne à maintes reprises que les sanctions 
prises à l'encontre du grand savant furent plutôt modérées ${ }^{40}$ et, d'autre part, que la condamnation n'émana pas de l'Église universelle mais de la simple Inquisition "a cui niuno tra $i$ teologi più zelanti ha mai accordato il diritto della infallibilità ${ }^{4 \mathrm{I}}$.

Après ces considérations biographiques, Tiraboschi, en un catalogue un peu fastidieux, passe en revue, preuves à l'appui en bon érudit, les inventions de Galilée (téléscope, microscope, pendule thermomètre, balance hydrostatique) comme autant de miracles dignes d'une hagiographie ${ }^{42}$. Cette tonalité est renforcée par le ton admiratif du texte et par le fait que presque aucun reproche ne vient ternir la gloire de Galilée, homme parfait, véritable héros. En un crescendo triomphal, Tiraboschi parle encore de ses découvertes "in ogni parte del regno della natura ${ }^{43}$ " qui sont autant d'illustrations éclatantes de sa supériorité sur les autres savants. Qu'il dépasse aussi par sa méthode d'étude, si préférable aux «inutili sottigliezze» de Francesco Patrizi, Girolamo Cardano ou Giordano Bruno mais aussi de René Descartes que Tiraboschi fustige vertement d'avoir osé manifester "un intollerabil disprezzo del Galileo", raison pour laquelle "il suo sistema [di Cartesio] omai è rimirato non altrimenti che un sogno e le osservazioni del Galileo al contrario [sono] riconosciute comunemente e adottate com principi su cui quasi tutta è fondata la moderna la filosofia ${ }^{44}$ ». Tiraboschi trouve tout de même en Galilée quelques excès, soulignés avec délicatesse et retenue. Ainsi, dans une polémique contre un de ses contradicteurs, le père Scheiner, à propos des taches solaires "scrisse dello Scheiner con tali espressioni che dal commercio dei dotti dovrebbono essere bandite». Et puis, "non ugualmente felice fu [...] nell'esaminar le comete 45 ".

La notice consacrée à Galilée s'achève sur un florilège d'éloges dont la tonalité est également héroïque. Il y a là bien encore une preuve que Galilée est vraiment "uno de' più rari geni che dalla natura siano stati prodotti». On remarque que les auteurs de ces éloges sont exclusivement des étrangers comme Leibniz, Bernoulli, Kepler, Newton, Keill. En effet, même si ce sont les Italiens, comme dit Tiraboschi dans sa préface, qui doivent connaître leurs héros, la caution des étrangers reste encore fondamentale car "meno sospetta» à un moment où chaque nation cherche à glorifier ses hommes illustres ${ }^{46}$.

\footnotetext{
40. Ibid., vol. VIII, p. I73-I75.

4I. Ibid., vol. VIII, p. I77.

42. Ibid., vol. VIII, p. I77-I85.

43. Ibid., vol. VIII, p. I89.

44. Ibid., vol. VIII, p. I89-I9o.

45. Ibid., vol. VIII, p. I93-I95.

46. Ibid., vol. VIII, p. 405.
} 
Voilà comment Tiraboschi nous guide dans la selva de la culture du $\mathrm{XVII}^{\mathrm{e}}$ siècle. Il nous fait ainsi découvrir un bon nombre d'hommes illustres par une série de notices plutôt austères et stéréotypées. Il nous met également en garde, non sans une certaine complaisance, anecdotique il est vrai, contre les faux héros. Il s'amuse et fait sourire le lecteur aux dépens de ceux dont «l'héroïsme» ne tient qu'à une caractéristique étrange, fustige ceux qui, par leur faute, ont mal utilisé leurs talents, s'enthousiasme enfin sur les figures vraiment héroïques comme Galilée. Bref, Tiraboschi définit un véritable panthéon mais aussi un enfer littéraire pour ce siècle que nous avons plus particulièrement étudié ici mais également pour tous les autres. Il propose ainsi, le premier, un classement, une typologie d'auteurs illustres qui synthétise et met en ordre les remarques et informations glanées dans un très grand nombre de sources précédentes, ce qui représente une avancée méthodologique importante. Même s'il est âprement critiqué par les intellectuels, en particulier romantiques, qui lui reprochent notamment sa pesante érudition et son absence de sensibilité littéraire ${ }^{47}$, il n'en reste pas moins que Tiraboschi reste, même négativement, une référence incontournable pour les critiques postérieurs. D’ailleurs, une bonne partie des jugements qu'il exprime sur les écrivains du XviI siècle, notamment l'opposition implicite entre l'hérö̈sme de Galilée et la corruption de Marino et plus généralement entre la décadence des lettres et l'essor de la science du temps sera reprise durablement, certes dans une perspective idéologique bien différente, dans les histoires de la littérature. 